\title{
Le bilan optimal avant TAVI
}

Pierre Lantelme, Brahim Harbaoui

Pierre Lantelme, MD,PhDa,b ${ }^{a}$ Brahim Harbaoui MD,PhD ${ }^{a, b}$

a Service de cardiologie, Hôpital Croix-Rousse et Hôpital Lyon Sud, Hospices Civils de Lyon, Lyon, France

b Université Lyon 1, CREATIS UMR5220; INSERM U1044; INSA-15 Lyon, France

Nombre de mots: 3300 mots

1 tableau; 2 Figures

Financement: Aucun

Conflit d'intérêt: Aucun

Correspondance: Pr Pierre Lantelme, Cardiology Department, Hôpital Croix-Rousse et Hôpital Lyon Sud, 103 Grande Rue de la Croix-Rousse, F-69004, Lyon, France. Email: pierre.lantelme@chu-lyon.fr

Fax: (33) 472071 674. Phone: (33) 472071668 


\title{
Le bilan optimal avant TAVI
}

\author{
Nombre de mots : 3300 mots \\ 1 tableau; 2 Figures
}

Financement: Aucun

Conflit d'intérêt: Aucun

\section{Résumé (250 mots max)}

Le nombre de procédures TAVI ne cesse d'augmenter chaque année en parallèle à l'élargissement des indications. Le succès procédural et à plus long terme passe par l'identification des patients susceptibles d'en bénéficier et par une préparation méticuleuse de la procédure lors du bilan pré-TAVI. Les objectifs de ce bilan sont multiples : validation de l'indication clinique, faisabilité de la procédure, sélections de la prothèse et de la voie d'abord et anticipation des difficultés techniques. Le scanner de la valve et des axes vasculaires est incontournable et représente la pierre angulaire de ce bilan. L'évaluation gériatrique garde toute sa place chez les patients âgés, fragiles, à haut risque ou contre indiqués à la chirurgie. L'évaluation des comorbidités cardiaques et extracardiaques est systématique et pourra être intégrée dans des scores de risques dédiés afin d'aider à la stratification du risque à moyen et long terme par la Heart Team.

\section{Abstract}


The number of TAVI procedures is rapidly increasing owing to the extension of indications. Procedural and long-term success warrant appropriate patient selection and rigorous preparation of the procedure during the pre-TAVI work-up. The aims of this work-up are several: validation of the indication, feasibility and safety of the procedure, selection of the prosthesis and of the route, anticipation of technical challenges. The CT-scan is the cornerstone of this assessment allowing for valve and vascular access appraisal. The geriatric evaluation remains important in the elderly, frail, high-risk patients. The assessment of cardiac and extracardiac comorbidities is systematic and may be incorporated into dedicated risk scores to improve the prediction of post-TAVI outcomes; this should be the basis of the decision-making process by the Heart Team.

Mots Clés: TAVI, rétrécissement aortique, scanner, accès vasculaires, complications, scores de risques, calcifications

Key words: TAVI, TAVR, aortic stenosis, CT scan, vascular access, complications, risk scores, calcifications

\section{Introduction : objectifs du bilan pré TAVI}

Le TAVI s'impose de plus en plus comme une technique de référence dans le traitement de la sténose aortique $(\mathrm{SA})$ serrée et symptomatique. Contrairement à la chirurgie classique, le TAVI n'a virtuellement aucune contre-indication et les progrès techniques sont tels que tout malade est quasiment accessible à cette technique. Pour autant il subsiste une morbidité du geste et tous les patients ne bénéficient pas 
forcément de l'intervention à moyen terme; ainsi jusqu'à $20 \%$ des patients traités par TAVI ont une évolution défavorable caractérisée par un décès avant un an ou par une insuffisance cardiaque résiduelle (1). Dans cette perspective l'évaluation préprocédurale - «le bilan pré-TAVI »- est essentielle pour que la procédure ne se réduise par un challenge technique mais constitue une option thérapeutique raisonnée susceptible d'améliorer la qualité de vie et la survie des patients. On peut ainsi identifier 3 objectifs majeurs de bilan pré-TAVI :

1) Valider l'indication ;

2) planifier l'intervention (voie d'abord, taille/type de la prothèse);

3) identifier des facteurs de vigilance ou de complications. Les complications cardiaques et vasculaires étant gravissimes, il est important de les anticiper. Dans beaucoup de cas, elles peuvent l'être par une évaluation rigoureuse et systématique de la situation. Le scanner est indiscutablement l'examen clé de ce bilan. II doit couvrir les troncs supra aortiques et l'ensemble de l'aorte jusqu'aux artères fémorales. II est réalisé en apnée avec synchronisation cardiaque en mode rétrospectif permettant de reconstruire les phases 40 et $75 \%$ du cycle cardiaque de façon à pouvoir analyser la valve et réaliser les mesures (sur la phase systolique, $40 \%$ ). Le kilovoltage et les mAs doivent être adaptés à chaque patient. L'injection de produit de contraste est biphasique avec $80 \mathrm{cc}$ de produit de contraste iodé suivi de $50 \mathrm{cc}$ de sérum physiologique à un débit de $3 \mathrm{ml} / \mathrm{seconde}$. Le débit d'injection doit être diminué en cas de SA très sévère pour ne pas risquer de complications liées à la surcharge hémodynamique induite. Une méthode de détection de bolus (type bolus tracking) est utilisée avec une région d'intérêt placée dans l'aorte descendante et un seuil à $130 \mathrm{UH}$. Sur les scanner le permettant, il est utile de stopper la 
synchronisation cardiaque en cours d'acquisition sur l'abdomen et le pelvis. Les images doivent être reconstruites en coupe de moins de $1 \mathrm{~mm}$. Le scanner est systématique et permet d'apporter des éléments techniques et pronostiques. Ces éléments, ainsi que ceux fournis par les autres examens qui seront détaillés, seront repris en réunion multidisplinaire (Heart Team) pour valider la solution thérapeutique, planifier la procédure et préciser les risques afin d'éclairer le patient et son entourage sur le bénéfice attendu de la prise en charge.

\section{Validation de l'indication}

Le TAVI est indiqué en 2019 en France chez des patients porteurs d'un RA serré symptomatique à risque chirurgical intermédiaire et élevé chez qui l'espérance de vie dépasse 1 an. L'étape de confirmation de la SA sévère est basée essentiellement sur l'échographie transthoracique (ETT) et ne sera pas reprise ici. L'utilisation de score de risque chirurgicaux tels que l'Euroscore I ou II, le STS score reste encore largement de mise pour identifier ces patients même si aucun d'entre eux n'est adapté à la pratique du TAVI. La prédiction de la survie à un an est plus délicate et pourtant essentielle pour éclairer la décision du patient et éviter une intervention futile. Les scores chirurgicaux ne sont d'aucune utilité en la matière $(2,3)$. En revanche, des facteurs de mauvais pronostic sont bien identifiés et peuvent se décomposer en facteurs non cardiaques et cardiaques (1). Parmi les facteurs non cardiaques on retient principalement l'altération de la fonction rénale, la présence d'une BPCO et à fortiori l'oxygéno-dépendance, l'existence de pathologies infectieuses (foyers dentaires) ou encore, une néoplasie sous-jacente (connue ou non). Ces comorbidités pourront être dépistés à l'occasion de ce bilan sur les examens biologiques (numération sanguine, $\mathrm{CRP}$ ), radiologiques 
(orthopantomogramme), à l'exploration fonctionnelle respiratoire ou au scanner systématique (4).

Dans cette perspective, l'évaluation de la fragilité est également importante et s'appuie sur l'identification des syndromes gériatriques (trouble cognitifs, troubles de l'humeur, malnutrition, chute...) et de leur retentissement fonctionnel ; elle s'intéresse également à la polymédication et à l'environnement psychosocial (5). Cette évaluation participe à part entière à la validation de l'indication afin de prédire les chances d'amélioration de la qualité de vie après TAVI ou au contraire le risque de séjour prolongé voire d'institutionnalisation après la procédure. Elle permet également de mieux préparer les patients à la procédure (correction d'une dénutrition, prévention du syndrome confusionnel...) et d'organiser le post-TAVI (mise en place d'aides telle que des aides ménagères et IDE à domicile etc). Des outils de dépistage de la fragilité (VES-13, grip test, Timed up and go test....) ont été proposé afin d'aider les praticiens à identifier les patients vulnérables (6). L'évaluation gériatrique doit autant que faire se peut avoir lieu avant les examens invasifs ou nécessitant l'injection de produit de contraste.

D'autres facteurs de mauvais pronostic concernent la sphère cardio-vasculaire ; on peut retenir la diminution de la fraction d'éjection systolique ventriculaire gauche, le bas gradient, I'HTAP et l'insuffisance mitrale sévère. L'échographie transthoracique (ETT) constitue l'examen de référence pour les identifier. Pour identifier une pathologie mitrale, le scanner apporte également des informations très importantes de par sa capacité à détecter des calcifications mitrales; l'importance des calcifications mitrales évaluées au scanner apparait comme un puissant prédicteur de mortalité à 1 an dans plusieurs séries (7). La charge calcique aortique évaluée au scanner représente également un puissant marqueur de décès et d'insuffisance 
cardiaque post-TAVI (8-10) probablement par l'impact qu'exerce une aorte rigide sur la post-charge du ventricule gauche. L'existence d'une maladie coronaire est fréquente (11) et son dépistage se fait par une coronarographie pré-TAVI systématique (ESC guidelines, classe $1 C$ ). La valeur pronostique des lésions coronaires est incertaine (12) et le bénéfice de la revascularisation également. Compte-tenu de l'ambiguïté angiographique et des difficultés de pratique des tests d'ischémie non invasifs dans cette population, l'utilisation de la physiologie coronaire est particulièrement attractive. Elle souffre cependant d'un problème de validation puisqu'aucune étude pronostique n'a été réalisée dans le contexte de SA avec les indices de pression avec ou sans hyperhémie. Or les facteurs de confusion sont nombreux notamment en rapport avec la modification des résistances microcirculatoires et la diminution de la CFR. Quelques études de portée limitée semblent montrer une relative stabilité des indices après TAVI ; Pesarini et al. ont montré une baisse de la FFR après TAVI sans le sous-groupe des patients avec une FFR $\leq 0.8$ (13). Dans l'attente de données plus formelles, il ne faut pas s'interdire l'utilisation de la FFR (ou de l'IFR) dans le RA en considérant qu'une lésion avec une FFR $\leq 0.8$ a toutes les chances de le rester après TAVI. II y'a en revanche une incertitude pour les lésions dont la FFR serait « borderline ».

Plusieurs scores intégrant certaines de ces variables ont été proposés comme par exemple le score dérivé de la cohorte Partner ou le score TARIS avec néanmoins une prédiction sub-optimale de la survie à moyen et long-terme (1). S'appuyant en partie sur la charge calcique au niveau de l'aorte thoracique mesurée au scanner (voir figure 1), le score CAPRI a été récemment proposé (2). Outre cette charge calcique aortique, le score CAPRI se compose de facteurs de morbidités classiques (âge, genre, fonction rénale, présence d'une pathologie pulmonaire obstructive) de 
facteurs de risque cardiaques (stade NYHA, fraction d'éjection, gradient transaortique, pressions pulmonaires) et également de facteurs de risque vasculaires. II est prédicteur de la mortalité cardio-vasculaire et totale à 1 an et s'avère très discriminant. II est également prédictif de la survenue d'une insuffisance cardiaque résiduelle (14). II représente actuellement le score pronostique dédié le plus validé dans le TAVI. Les examens utiles à cette première étape son résumés dans le tableau.

\section{Voie d'abord}

La navigabilité de la prothèse est un élément déterminant de la faisabilité du TAVI. Le scanner est l'examen clé pour identifier des difficultés éventuelles à l'utilisation de la voie transfémorale (15) qui est la voie élective. Les facteurs pouvant obérer une voie fémorale ou représenter un point de vigilance particulier sont représentés par des calcifications importantes notamment en regard de la zone de ponction surtout si elles sont antérieures ou si il existe une bifurcation très haute. Un diamètre minimal au niveau de l'axe ilio-fémoral principal de 5 à $6 \mathrm{~mm}$ est exigé selon le type de prothèse. Lors de cette analyse, il est important d'évaluer également la tortuosité des axes qui peut également gêner la navigabilité de la prothèse et nécessiter l'utilisation de guides plus rigides ou l'utilisation d'introducteurs. L'étude de la navigabilité se poursuit au niveau de l'aorte avec la recherche de plaques inhomogènes voir thrombotiques, de zones anévrysmales ou encore de tortuosités très marquées notamment au niveau de l'arche («gothic arch). Enfin les artères carotides et sousclavières seront évalués en cas d'accès fémoral défavorable ; un examen des axes sous-claviers peut être intéressant avec le développement de la voie radiale comme accès secondaire et avec le développement des systèmes de protection cérébraux mis en place par voie radiale. En cas d'impossibilité de passage par les accès 
périphériques ce qui devient rare, une étude des rapports anatomiques en vue d'une voie transaortique ou transapicale se fera au cas par cas.

Le doppler des TSA peut représenter un complément intéressant pour préciser le risque embolique en cas de recours à une voie transcarotidienne.

Certaines équipes profitent de la coronarographie systématique faite dans le cadre du bilan (cf $\S$ validation de l'indication) pour faire une angiographie du carrefour aortique et préciser la voie d'abord mais la pratique la plus habituelle est d'évaluer ces voies d'abord sur le scanner. Les examens utiles à cette étape son résumés dans le tableau.

\section{Sélection de la prothèse}

Le scanner occupe, là encore, une place majeure. II permet de confirmer les caractéristiques anatomiques de la valve (bi- ou tricuspide), de préciser la charge calcique valvulaire et annulaire ainsi que la présence de "calcifications tueuses " comportant un risque accru de perforation, toutes ces caractéristiques étant prises en compte dans le choix du type de prothèse («balloon expandable » versus "selfexpandable »). Ces caractéristiques ainsi que l'angulation aortique seront également prises en compte pour le recours éventuel à une prédilatation. Le scanner est l'examen clé pour déterminer la taille de la prothèse en identifiant l'anneau virtuel ; il correspond au plan d'alignement des nadirs des 3 cusps aortiques. La taille de la prothèse sera précisée en fonction de la surface ou du périmètre ainsi que des diamètres minimum et maximum de cet anneau virtuel, de façon manuelle ou automatique grâce à des logiciels dédiés (Endosize, 3mensio ...). Sont également mesurés, la hauteur et la profondeur des sinus de Valsalva (encombrement de la prothèse), le diamètre de la jonction sino-tubulaire (pour les prothèses de type 
Evolute). Enfin, le scanner permet la détermination de l'incidence de travail permettant ainsi un gain de temps, d'irradiation et de produit de contraste lors de l'implantation (16). Les examens utiles à cette étape son résumés dans le tableau.

\section{Stratification du risque procédural}

Le risque procédural dépend de la complexité vasculaire (cf $\S$ voie d'abord), de l'interaction valve/coronaire, de la présence spécifique de calcifications « tueuses » et enfin du risque rythmique. Une attention particulière sera également portée à la taille du ventricule gauche (VG) (ETT et Scanner) afin de prévoir l'utilisation de guides intra-VG adaptés à son anatomie. Bien entendu le risque rénal inhérent à toute injection de contraste doit conduire à une vigilance chez les patients insuffisants rénaux ainsi que les troubles de crase sanguine.

Le risque coronaire per-procédural essentiel est la bascule de matériel athérothrombotique ou valvulaire sur les ostia coronaires. Le scanner coronaire est une fois encore l'examen fondamental pour évaluer ce risque en permettant d'analyser la hauteur de naissance des ostia coronaires (distance anneau aortique virtuel et ostia) et le rapport avec la cusp aortique correspondante. Dans cette optique la profondeur des sinus de valsalva doit aussi être appréciée. En cas d'anatomie défavorable et sans autre alternative cela conduira à envisager une protection coronaire par guide +/- un « chimney stenting » lors de l'intervention (17).

Les troubles conductifs post TAVI nécessitant l'implantation d'un pacemaker représentent une des complications les plus fréquentes malgré l'expérience des opérateurs et les avancées technologiques (18). L'ECG pré-TAVI est capital pour évaluer le risque de troubles conductifs peri-procéduraux. Les anomalies ECG préTAVI les plus prédictives de BAV post-TAVI sont le bloc de branche droite (BBD) et à 
un moindre degré le PR long. Le scanner permet aussi d'étudier les coulées calcaires septales s'étendant à l'anneau mitral qui sont également à risque de trouble de conduction de haut degré. La présence d'une FA est fréquente et donnera lieu à une gestion particulière du traitement anti-thrombotique. L'identification de ces différents risques doit conduire à une réponse adaptée en terme d'abord vasculaire, d'utilisation d'introducteurs, de type de valve, de système de protection illustré dans la figure 2. La balance bénéfice/risque doit toujours être réévaluée en présence de plusieurs facteurs de complexité.

\section{Synthèse : la Heart-Team}

Le bilan pré TAVI doit permettre de répondre à un certain nombre de questions essentielles pour la confirmation de l'indication, de la faisabilité, de la planification et du bon déroulement de la procédure. Ce bilan doit permettre d'identifier les difficultés particulières afin de prévenir la morbi-mortalité péri procédurale. Afin d'améliorer l'objectivité de la décision, il est essentiel que cette décision soit partagée afin d'en améliorer l'objectivité. Dans cette perspective, la multiplicité des expertises au sein de la Heart Team est essentielle et doit rester le pilier de la prise en charge de ces patients souvent complexes afin d'optimiser la pertinence de la prise en charge.

\section{Conclusions}

Le bilan pré-TAVI est un temps essentiel de la prise en charge des patients porteurs d'une SA serrée potentiellement éligibles à un TAVI. Le scanner pré-TAVI s'est imposé comme un examen essentiel qui continue de nous fournir toujours plus d'informations sur les chances de succès procédural à court et long terme. L'évaluation clinique reste néanmoins toujours aussi essentielle dans cette population fragile. On peut espérer que l'arrivée de nouveaux scores de risque 
dédiés permettra d'aider la décision thérapeutique et la sélection des bons candidats à cette technique.

\section{Légendes des Figures}

Figure 1 : exemple de scanner montrant en reconstruction 3D et en coupe une aorte thoracique A) peu, B) modérément, C) très calcifiée. La charge calcique aortique est très prédictive de la survie post-TAVI; elle constitue une variable constitutive du score CAPRI.

Figure 2 : principaux facteurs de risque procéduraux lors d'un TAVI et options qui en découlent. Le cumul de facteurs de risque procéduraux accroit le risque procédural.

\section{Bibliographie}

1. Puri R, lung B, Cohen DJ, Rodes-Cabau J. TAVI or No TAVI: identifying patients unlikely to benefit from transcatheter aortic valve implantation. European heart journal 2016;37:2217-25.

2. Lantelme P, Eltchaninoff $H$, Rabilloud M et al. Development of a Risk Score Based on Aortic Calcification to Predict 1-Year Mortality After Transcatheter Aortic Valve Replacement. JACC Cardiovascular imaging 2019;12:123-132.

3. Ludman PF, Moat N, de Belder MA et al. Transcatheter aortic valve implantation in the United Kingdom: temporal trends, predictors of outcome, and 6-year follow-up: a report from the UK Transcatheter Aortic Valve Implantation (TAVI) Registry, 2007 to 2012. Circulation 2015;131:1181-90.

4. Fathala A, Bin Saeedan M, Zulfiqar A, Al Sergani H. Non-Cardiovascular Computed Tomography Incidental Findings in Patients Who Underwent Transaortic Valve Implantation Procedure. Cardiology research 2017;8:13-19.

5. Rogers $\mathrm{T}$, Alraies MC, Moussa Pacha $\mathrm{H}$ et al. Clinical Frailty as an Outcome Predictor After Transcatheter Aortic Valve Implantation. The American journal of cardiology 2018;121:850-855.

6. Anand A, Harley C, Visvanathan A et al. The relationship between preoperative frailty and outcomes following transcatheter aortic valve implantation: a systematic review and meta-analysis. European heart journal Quality of care \& clinical outcomes 2017;3:123-132.

7. Abramowitz $\mathrm{Y}$, Kazuno $\mathrm{Y}$, Chakravarty $\mathrm{T}$ et al. Concomitant mitral annular calcification and severe aortic stenosis: prevalence, characteristics and outcome 
following transcatheter aortic valve replacement. European heart journal 2017;38:1194-1203.

8. Harbaoui B, Courand PY, Charles $P$ et al. Aortic calcifications present the next challenge after TAVR. Journal of the American College of Cardiology 2015;65:105860.

9. Harbaoui B, Montoy M, Charles $\mathrm{P}$ et al. Aorta calcification burden: Towards an integrative predictor of cardiac outcome after transcatheter aortic valve implantation. Atherosclerosis 2016;246:161-8.

10. Gegeneva T, Vollema EM, Abou R et al. Prognostic Value of Thoracic Aorta Calcification Burden in Patients Treated With TAVR. JACC Cardiovascular imaging 2019;12:216-217.

11. Faroux L, Guimaraes L, Wintzer-Wehekind $\mathrm{J}$ et al. Coronary Artery Disease and Transcatheter Aortic Valve Replacement: JACC State-of-the-Art Review. Journal of the American College of Cardiology 2019;74:362-372.

12. Danson E, Hansen P, Sen S, Davies J, Meredith I, Bhindi R. Assessment, treatment, and prognostic implications of CAD in patients undergoing TAVI. Nature reviews Cardiology 2016;13:276-85.

13. Pesarini G, Scarsini R, Zivelonghi C et al. Functional Assessment of Coronary Artery Disease in Patients Undergoing Transcatheter Aortic Valve Implantation: Influence of Pressure Overload on the Evaluation of Lesions Severity. Circulation Cardiovascular interventions 2016;9.

14. Harbaoui B, Durand E, Dupre M et al. Significance of the CAPRI risk score to predict heart failure hospitalization post-TAVI: The CAPRI-HF study. International journal of cardiology 2019.

15. Wiegerinck EM, Marquering HA, Oldenburger NY et al. Imaging for approach selection of TAVI: assessment of the aorto-iliac tract diameter by computed tomography-angiography versus projection angiography. The international journal of cardiovascular imaging 2014;30:399-405.

16. Hell MM, Biburger L, Marwan $M$ et al. Prediction of fluoroscopic angulations for transcatheter aortic valve implantation by CT angiography: influence on procedural parameters. European heart journal cardiovascular Imaging 2017;18:906-914.

17. Spaziano M, Akodad M, Hovasse T, Lefevre T, Bouvier E, Chevalier B. Simultaneous TAVR and Left Main "Chimney" Stenting in a Patient With Low Left Main Height. JACC Cardiovascular interventions 2017;10:e185-e187.

18. Nazif TM, Dizon JM, Hahn RT et al. Predictors and clinical outcomes of permanent pacemaker implantation after transcatheter aortic valve replacement: the PARTNER (Placement of AoRtic TraNscathetER Valves) trial and registry. JACC Cardiovascular interventions 2015;8:60-9. 


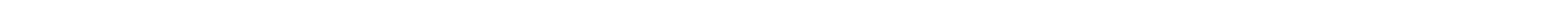




\section{Vasculaire}

- Tortuosités

- Calcifications +++

- Plaques +++

- Axes étroits

\section{Facteurs de risque}

\section{Aortique}

- Tortuosité (arche+++)

- Plaques +++

- Anévrisme

\section{Valvulaire}

- Calcifications +++

- Bicuspidie

- Aorte horizontale

- Valve-in-valve

- Faible distance anneau/ ostia coronaires $\varnothing$ facteur de risque Risque PROCEDURAL Faible: type $A$
- Voie fémorale

- Tous dispositifs

- Direct TAVI

-Voie secondaire radiale possible
1 facteur de risque Risque PROCEDURAL Intermédiaire: type $B$

- Voie alternative

- Introducteurs

-Dispositifs adaptés (franchissement, calcifications, embols)

-Prédilatation

-Protection coronaire

-Protection cérébrale ?
$>1$ facteurs de risque Risque PROCEDURAL Elevé: type $C$

- Idem risque intermédiaire

-Réévaluation balance bénéfice(comorbidités) /risque

- Opérateurs experts

-Stand-by chirugical ? 
Tableau : Objectifs du bilan pré-TAVI et examens correspondants

\begin{tabular}{|l|l|l|l|l|}
\hline & $\begin{array}{c}\text { Validation de } \\
\text { l'indication }\end{array}$ & Voie d'abord & $\begin{array}{c}\text { Sélection de } \\
\text { la prothèse }\end{array}$ & $\begin{array}{c}\text { Stratification du } \\
\text { risque }\end{array}$ \\
\hline Scanner aortique & & & & \\
\hline ETT & & & & \\
\hline Coronarographie & & & & \\
\hline Avis gériatrique & & & & \\
\hline EFR & & & & \\
\hline Doppler TSA & & & & \\
\hline ECG & & & & \\
\hline $\begin{array}{l}\text { Clairance de } \\
\text { creatinine }\end{array}$ & & & & \\
\hline $\begin{array}{l}\text { Numération } \\
\text { sanguine }\end{array}$ & & & & \\
\hline Coagulation & & & \\
\hline CRP & & & \\
\hline (pronostic à 1 an) & & & & \\
\hline varoscore (TAVI & & & & \\
\hline
\end{tabular}

ETT : échographie transthoracique ; EFR, exploration fonctionnelle respiratoire ; TSA, troncs supra-aortiques ; CRP,C reactive protein. 